\title{
Equilibrium States for a Plane Incompressible Perfect Fluid
}

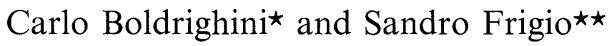 \\ Istituto Matematico, Università di Camerino, Camerino, Italy
}

\begin{abstract}
We associate to the plane incompressible Euler equation with periodic conditions the corresponding Hopf equation, as an equation for measures on the space of solenoidal distributions. We define equilibrium states as the solutions of the stationary Hopf equation. We find a class of equilibrium states which corresponds to a class of infinitely divisible distributions, and investigate the properties of gaussian and poissonian states. Equilibrium dynamics for a class of poissonian states is constructed by means of the Onsager vortex equations.
\end{abstract}

\section{Introduction}

The purpose of this paper is to exhibit a class of equilibrium states for a plane fluid which moves according to the incompressible Euler equation, and to study their main properties. Our treatment will be limited to the case of periodic boundary conditions, which allows an explicit use of Fourier methods.

Equilibrium states for the plane incompressible Euler fluid have been studied by physicists for a long time. Among the most significant contributions we may mention a paper by Lee [1] in which a class of gaussian states were introduced as macrocanonical equilibrium states corresponding to energy and enstrophy conservation, and a paper by Novikov [2] in which equilibrium states corresponding to poissonian distribution of vortices are studied.

The mathematical definition of equilibrium state which we give is based on the Hopf equation associated to the plane incompressible Euler equation, namely we define equilibrium states as solutions of the stationary Hopf equation. We recall that the Hopf equation describes the evolution of measures on phase space associated to the point evolution, and is written in terms of the characteristic functionals.

* Research partially supported by C.N.R., G.N.F.M.

$\star \star \quad$ C.N.R. fellowship, G.N.F.M. 
The Hopf equation plays here a role similar to that of the B-B-K-G-Y hierarchy equations in statistical mechanics (which is not surprising since the latter is nothing else than the evolution equation for the generating functional). In fact, although in mechanics there is an independent notion of equilibrium state (that of Gibbs state), it turns out that the class of Gibbs states corresponds to the class of stationary solutions of the B-B-K-G-Y hierarchy equations [3].

Our main result consists in exhibiting solutions of the stationary Hopf equation which are associated to the law of vorticity conservation along the trajectory of fluid particles, which is a characteristic feature of the plane Euler fluid. The corresponding measures are weak limits of some natural lattice measures with "Gibbs factor" associated to the constants of the motion of the Euler flow, energy excluded. Such equilibrium states are in one to one correspondence with a family of infinitely divisible distribution laws, and are characterized by the fact that vorticity is distributed as a generalized random field with independent values at each point. The vorticity distribution can be interpreted as a superposition of a gaussian distribution and a finite or infinite number of Poisson distributions.

We give an analysis of the main properties of the gaussian and poissonian states: we establish in particular that square summable velocity fields have zero measure in both cases. We also construct a further class of gaussian equilibrium states, associated to energy and enstrophy conservation, which are just the equilibrium states introduced by Lee [1]. They turn out to be absolutely continuous with respect to the gaussian measures previously considered.

If an equilibrium state is physically significant, it should be possible to show that it is the limit of the evolution of some class of physically reasonable states. This problem is similar to that of founding the Gibbs postulate in statistical mechanics (see for example [4]) and is probably of comparable mathematical difficulty. There are physical grounds which suggest that such a problem is reasonable at least for poissonian states (cf. [2]), and for gaussian states (see for instance [5] where results of computer experiments are provided, and references therein). In order to formulate the problem at a mathematical level one should first construct "nonequilibrium dynamics", which in our case amounts to extending the existence theorem for the Euler equation to a set of initial data which is large enough to contain the support of the states the evolution of which is to be studied (including equilibrium states). In statistical mechanics this problem has been solved only for one-dimensional and two-dimensional system $[6,7]$. In our case, we believe that one should, for the moment, be content with the construction of "equilibrium dynamics", that is, of time evolution for a set of full measure with respect to a fixed equilibrium state (in statistical mechanics this problem has already found a satisfactory solution, see [8] and references therein). We succeeded in constructing equilibrium dynamics for a class of poissonian equilibrium states, by showing that the solution of the Onsager vortex equations gives a (generalized) solution of the Euler equation.

The plan of the paper is the following. In Sect. 2 we expose the main facts on the plane Euler equation, with particular attention to the periodic case. In Sect. 3 we introduce the Hopf equation in a way which is convenient for measures on generalized function spaces (usually it is defined for measures on spaces of square 
summable functions, which are too small for our purposed). In Sect. 4 we introduce the main class of equilibrium states, and show than they are solutions of the stationary Hopf equation. In Sect. 5 we study gaussian and poissonian equilibrium states and construct gaussian equilibrium states associated to energy and enstrophy conservation. In Sect. 6 we construct the equilibrium dynamics for a class of poissonian states. Section 7 is devoted to a conclusive discussion.

\section{The Plane Incompressible Euler Equation}

\section{a. Generalities}

Let $\Omega$ denote an open set of the plane, and $\partial \Omega$ its boundary, which we suppose at least of class $C^{1}$. The plane incompressible Euler equation for the velocity field $\mathbf{u}(\mathbf{x}, t)=\left(u_{1}(\mathbf{x}, t), u_{2}(\mathbf{x}, t)\right)$ and the pressure field $p(\mathbf{x}, t),\left((\mathbf{x}, t) \in \Omega \times \mathbb{R}^{1}\right)$, in absence of external forces, is

$$
\begin{aligned}
\frac{\partial}{\partial t} \mathbf{u}+(\mathbf{u} \cdot \nabla) \mathbf{u} & =-\nabla p \\
\operatorname{div} \mathbf{u} & =0
\end{aligned}
$$

$\left[\boldsymbol{\nabla}=\left(\frac{\partial}{\partial x_{1}}, \frac{\partial}{\partial x_{2}}\right)\right.$ is the gradient, and $\left.\operatorname{div} \mathbf{u}=\sum_{i=1}^{2} \frac{\partial}{\partial x_{i}} u_{i}\right]$. Equations (2.1) and (2.2) are accompanied by the initial condition

$$
\mathbf{u}(\mathbf{x}, 0)=\mathbf{u}_{0}(\mathbf{x})
$$

and the boundary condition

$$
\left.\mathbf{u} \cdot \mathbf{n}\right|_{\partial \Omega}=0
$$

$\mathbf{n}$ being the outer normal on $\partial \Omega$. We recall that the solenoidality condition $(2.2)$ expresses incompressibility, and the boundary condition (2.4) means that the liquid cannot flow out of $\Omega$.

Equations (2.1) and (2.2) can be written in terms of the vorticity field $\omega(\mathbf{x}, t)$

$$
\omega(\mathbf{x}, t) \equiv \operatorname{rot} \mathbf{u}(\mathbf{x}, t) \equiv \frac{\partial u_{2}}{\partial x_{1}}-\frac{\partial u_{1}}{\partial x_{2}}
$$

( $\omega$ is a scalar in dimension 2). Defining, following Kato [9], the rotation of a scalar field $\phi(\mathbf{x})$ as the vector

$$
\operatorname{rot} \phi \equiv\left(\frac{\partial \phi}{\partial x_{2}},-\frac{\partial \phi}{\partial x_{1}}\right)
$$

we have

$$
\operatorname{rot} \operatorname{rot} \phi=-\Delta \phi \text {. }
$$

It is easily seen that a differentiable solenoidal vector field in $\Omega, \mathbf{v}(\mathbf{x})$, satisfying the boundary condition $\left.\mathbf{v} \cdot \mathbf{n}\right|_{\partial \Omega}=0$ can be uniquely reconstructed in terms of its rotation:

$$
\mathbf{v}(\mathbf{x})=\operatorname{rot} G(\operatorname{rot} \mathbf{v})
$$


$G$ being the inverse of $(-\Delta)$ with zero boundary conditions on $\partial \Omega$ [i.e. $G(\phi)$ is the solution of the equation $\left.\Delta \psi=-\phi,\left.\psi\right|_{\partial \Omega}=0\right]$.

In place of Eqs. (2.1) and (2.2) we can write

$$
\begin{aligned}
\frac{\partial \omega}{\partial t}+(\mathbf{u} \cdot \boldsymbol{\nabla}) \omega & =0 \\
\mathbf{u} & =\operatorname{rot} G(\omega) .
\end{aligned}
$$

Equation (2.8) expresses the law of "vorticity conservation for any fluid particle", i.e. it says that the "total derivative" of the vorticity is zero.

\section{b. Existence, Uniqueness and Constants of the Motion}

Existence, uniqueness [with $p(\mathbf{x}, t)$ determined up to an arbitrary function of time] and regularity for solutions of the problem (2.1)-(2.4) have been proved in various frameworks. For classical solutions (i.e. such that all the derivatives involved exist and are continuous), it is assumed that $\partial \Omega$ is at least of class $C^{2+\delta},(\delta>0)$ and the main tool of the proof is Schauder's fixed point theorem (see for example [9]). For "weak solutions", [i.e. solutions which are not necessarily differentiable and satisfy (2.1) and (2.2) in a "weak sense", that is, in some space of functionals] an existence and uniqueness result has been proved under the assumption that $\mathbf{u}_{0}$ is a square summable function with square summable gradient, and that $\partial \Omega$ is of class $C^{2}$ (cf. [10]). The proof is based on compactness arguments, and the solution is a continuous function of $t$ with values in the space of square summable vector functions $\left(L^{2}(\Omega)\right)^{2}$. All results for classical as well as for weak solutions hold for all times.

We now recall some well-known properties of the Euler equations. (We limit our considerations to classical solutions only.)

By "constant of the motion" we mean a functional of $\mathbf{u}$, which is unchanged as $\mathbf{u}$ evolves according to the Euler equation. We have:

Proposition 2.1. The energy

$$
E(t)=\frac{1}{2} \int_{\Omega}|\mathbf{u}(\mathbf{x}, t)|^{2} d x
$$

does not depend on $t$ if $\mathbf{u}(\mathbf{x}, t)$ is a classical solution of the problem (2.1)-(2.4) (by " $d x$ " we denote the Lebesgue measure on $\mathbb{R}^{2}$ ).

Proof. From the existence and uniqueness theorem for classical solutions we have that $E(t)$ exists and is differentiable in $t$ for any $t$. We have

$$
\frac{d}{d t} E(t)=-\int_{\Omega} \mathbf{u} \cdot(\mathbf{u} \cdot \nabla) \mathbf{u} d x=-\int_{\Omega}(\mathbf{u} \cdot \nabla) \frac{1}{2}|\mathbf{u}|^{2} d x=-\frac{1}{2} \int_{\partial \Omega}|\mathbf{u}|^{2} \mathbf{u} \cdot \mathbf{n} d x=0 .
$$

There are many other constants of the motion, as a consequence of the "law of vorticity conservation" (2.8):

Proposition 2.2. For any continuous function $f \in C(\mathbb{R})$ the functional

$$
I_{f}=\int_{\Omega} f(\omega(\mathbf{x}, t) d x
$$

is a constant of the motion if $\mathbf{u}$ is a classical solution of problem (2.1)-(2.4). 
Proof. If $f \in C^{1}(\mathbb{R})$, so that $I_{f}$ is differentiable, we have

$$
\begin{aligned}
\frac{d}{d t} I_{f} & =\int_{\Omega} f^{\prime}(\omega(\mathbf{x}, t)) \frac{\partial}{\partial t} \omega(\mathbf{x}, t) d x \\
& =-\int_{\Omega} f^{\prime}(\omega)(\mathbf{u} \cdot \boldsymbol{\nabla}) \omega d x=-\int_{\Omega}(\boldsymbol{\nabla} f(\omega) \cdot \mathbf{u}) d x \\
& =-\int_{\partial \Omega} f(\omega)(\mathbf{u} \cdot \mathbf{n}) d x+\int_{\Omega} f(\omega) \operatorname{div} \mathbf{u} d x=0 ;
\end{aligned}
$$

so that the result is true for $f \in C^{1}$. For $f \in C$ it can be obtained by a density argument.

A particularly important role in physics plays "enstrophy", that is

$$
S=\frac{1}{2} \int_{\Omega}|\omega(\mathbf{x}, t)|^{2} d x \text {. }
$$

\section{c. The Euler Equation on the Torus. Finite Dimensional Approximations}

We are interested in the case in which the boundary condition (2.3) is replaced by periodic conditions. More precisely, we consider (2.1) and (2.2) on a flat torus $T^{2}$ which we identify with a square of side $2 \pi$, with opposite sides glued together: $T^{2}=[0,2 \pi] \times[0,2 \pi] \bmod 2 \pi . \mathbf{u}(\mathbf{x}, t)$ may be determined only up to an arbitrary constant, which we fix by imposing the following "zero average" condition

$$
\int_{T^{2}} \mathbf{u}(\mathbf{x}, t) d x=0
$$

It would not be hard to extend to this case the results on existence, uniqueness and regularity described in Sect. 2a. However by an explicit use of Fourier transform it is possible to give a constructive proof of existence and uniqueness, which we shall presently outline.

Consider the Fourier expansion of $\mathbf{u}(\mathbf{x}, t)$

$$
\mathbf{u}(\mathbf{x}, t)=\frac{1}{2 \pi} \sum_{\mathbf{k} \in \overline{\mathbb{Z}}^{2}} \exp (i \mathbf{k} \cdot \mathbf{x}) \hat{u}_{\mathbf{k}}(t) \frac{\mathbf{k}^{\perp}}{k}
$$

[here $\overline{\mathbb{Z}}^{2}=\mathbb{Z}^{2} \backslash(0,0), \mathbb{Z}^{2}$ being the plane integer lattice, $\mathbf{k}^{\perp} \in \mathbb{Z}^{2}$ is obtained by $\mathbf{k} \equiv\left(k_{1}, k_{2}\right)$ by setting $\mathbf{k}^{\perp}=\left(k_{2},-k_{1}\right)$, and $\left.k=|\mathbf{k}|\right]$. The Fourier components of $\mathbf{u}$ are proportional to $\mathbf{k}^{\perp}$ as a consequence of (2.2). Moreover, since $\mathbf{u}$ is real we have

$$
\hat{u}_{\mathbf{k}}=-\overline{\hat{u}}_{-\mathbf{k}} \text {. }
$$

By formal substitution we get the Euler equations in Fourier form

$$
\dot{\hat{u}}_{\mathbf{k}}(t)=-i \sum_{\substack{\mathbf{h}+\mathbf{m}=\mathbf{k} \\ \mathbf{h}, \mathbf{m} \in \overline{\mathbb{Z}}^{2}}} \Gamma_{\mathbf{h m k}} \hat{u}_{\mathbf{h}}(t) \hat{u}_{\mathbf{m}}(t) \quad\left(\mathbf{k} \in \overline{\mathbb{Z}}^{2}\right)
$$

with $\Gamma_{\mathbf{h m k}}=\left(\mathbf{h}^{\perp} \cdot \mathbf{m}\right)\left(h^{2}-m^{2}\right) / h m k$.

The initial condition (2.3) becomes

$$
\hat{u}_{\mathbf{k}}(0)=1 /\left[(2 \pi)^{2} k\right] \int_{T^{2}} \mathbf{k}^{\perp} \cdot \mathbf{u}_{0}(\mathbf{x}) \exp (-i \mathbf{k} \cdot \mathbf{x}) d x \equiv \hat{u}_{\mathbf{k}}^{(0)}
$$


The Fourier components of $p(\mathbf{x}, t)$ are given by

$$
p_{\mathbf{k}}(t)=-i \sum_{\substack{\mathbf{h}+\mathbf{m}=\mathbf{k} \\ \mathbf{h}, \mathbf{m} \in \overline{\mathbb{Z}}^{2}}}\left[\left(\mathbf{h}^{\perp} \cdot \mathbf{m}\right)\left(\mathbf{h} \cdot \mathbf{m}^{\perp}\right) / h m k\right] \hat{u}_{\mathbf{h}} \hat{u}_{\mathbf{m}} \quad\left(\mathbf{k} \in \overline{\mathbb{Z}}^{2}\right)
$$

so that the problem reduces to solving the system of infinite coupled ordinary differential equations (2.12) subject to the initial conditions (2.13). It is natural here to apply the method of finite dimensional approximants ("Faedo-Galerkin" method).

Definition 2.1. For any set $I \subset \overline{\mathbb{Z}}^{2}$ such that

i) $I=-I \equiv\left\{\mathbf{k} \in \overline{\mathbb{Z}}^{2} \mid-\mathbf{k} \in I\right\}$

ii) for any $\mathbf{k} \in I$ there is at least a pair $\mathbf{h}, \mathbf{m} \in I$ such that $\mathbf{k}=\mathbf{h}+\mathbf{m}$,

we define the corresponding finite dimensional approximant (hereafter f.d.a.) as the system of ordinary differential equations

$$
\begin{aligned}
& \dot{u}_{\mathbf{k}}^{(I)}(t)=-i \sum_{\substack{\mathbf{h}+\mathbf{m}=\mathbf{k} \\
\mathbf{h}, \mathbf{m} \in I}} \Gamma_{\mathbf{h m m}} u_{\mathbf{h}}^{(I)}(t) u_{\mathbf{m}}^{(I)}(t) \\
& u_{\mathbf{k}}^{(I)}(0)=\hat{u}_{\mathbf{k}}^{(0)} .
\end{aligned}
$$

Since we have

$$
\Gamma_{\mathbf{h m k}}+\Gamma_{\mathbf{k h m}}+\Gamma_{\mathbf{m k h}}=k^{2} \Gamma_{\mathbf{h m k}}+m^{2} \Gamma_{\mathbf{k h m}}+h^{2} \Gamma_{\mathbf{m k h}}=0
$$

it is easily seen that

$$
\begin{aligned}
& E^{(I)}=\frac{1}{2} \sum_{\mathbf{k} \in I}\left|u_{\mathbf{k}}^{(I)}\right|^{2} \\
& S^{(I)}=\frac{1}{2} \sum_{\mathbf{k} \in I} k^{2}\left|u_{\mathbf{k}}^{(I)}\right|^{2}
\end{aligned}
$$

are constant in time. This in its turn implies an existence and uniqueness theorem for the problem (2.15) and (2.16).

A constructive existence and uniqueness theorem for the problem (2.12) and (2.13) can be obtained along the following lines (cf. [11]). Suppose that $\sum k^{2}\left|\hat{u}_{\mathbf{k}}^{(0)}\right|^{2}<\infty$, and consider the sequence $\left\{u^{(N)}(t)\right\}_{N=1}^{\infty}$ of solutions of equations (2.15) and (2.16) corresponding to the index sets $I_{N}=\left\{\mathbf{k} \in \overline{\mathbb{Z}}^{2}|| \mathbf{k} \mid \leqq N\right\}$. The sequence converges in $l_{2}$, as $N \rightarrow \infty$, uniformly for $t$ in any finite interval. It follows that the corresponding sequence of Fourier antitrasforms converges in the space of square summable solenoidal functions on $T^{2}$ satisfying condition $\left(2.4^{\prime}\right), \mathscr{L}_{2}$ (which is a closed subspace of $\left[L^{2}\left(T^{2}\right)\right]^{2}$ ). The limit satisfies (in weak sense) the Euler equation (2.1) and (2.2), which is equivalent to the Eqs. (2.12) and (2.14) and is unique within the class of functions with square summable gradient. Moreover it is possible to estimate the error which is done by taking the $N$-th function of the sequence instead of the true solution. (We believe that a similar constructive theorem holds in the case of a domain with boundary as well, although it might be difficult to carry out the proof because of the inconvenience of the explicit Fourier representation.) 
It is not hard to see that for periodic boundary conditions equations (2.6)-(2.9) hold unchanged, [if by $G$ we understand the inverse of $(-\Delta)$ with periodic boundary conditions], as well as Propositions 2.1 and 2.2.

Notice that although f.d.a.'s for some particular index set $I$ may possess other constants of the motion independent of $E^{(I)}$ and $S^{(I)}$, there is evidence that there are no such additional invariants which are common to an infinite sequence of f.d.a.'s corresponding to the index sets $I_{N}[12]$.

\section{The Hopf Equation}

Periodic conditions are convenient because it is easy to work in Fourier representation. We begin by introducing it.

Let $\mathscr{S}\left(T^{2}\right)$ be the linear space of infinitely differentiable real functions with zero mean [i.e. such that $\int_{T^{2}} \phi(\mathbf{x}) d x=0, \forall \phi \in \mathscr{S}\left(T^{2}\right)$ ] ("test functions"), endowed with the usual Schwartz topology generated by the seminorms $\|\cdot\|_{k_{1}, k_{2}}$ $\left(k_{1}, k_{2}=0,1, \ldots\right)$ :

$$
\|\phi\|_{k_{1}, k_{2}}=\max _{\mathbf{k} \in T^{2}}\left|\frac{\partial^{k_{1}+k_{2}}}{\partial x_{1}^{k_{1}} \partial x_{2}^{k_{2}}} \phi\right| .
$$

Consider the subspace of $\mathscr{S}\left(T^{2}\right) \times \mathscr{S}\left(T^{2}\right)$ consisting of all solenoidal vector functions, which is closed in the product topology, and which we shall denote by $\tilde{\mathscr{S}}\left(T^{2}\right) . \tilde{\mathscr{S}}\left(T^{2}\right)$ is of course a locally convex space, and we may take on the family of seminorms $\|\cdot\|_{n}(n=0,1, \ldots)$ :

$$
\|\underline{\theta}\|_{n}=\left(\operatorname{rot} \underline{\theta},(-\Delta)^{n-1} \operatorname{rot} \underline{\theta}\right)^{1 / 2}
$$

$(\cdot, \cdot)$ being the usual scalar product in $L^{2}\left(T^{2}\right)$.

To any $\underline{\theta} \in \tilde{\mathscr{S}}\left(T^{2}\right)$ its Fourier expansion [cf. (2.11)] associates a scalar sequence $\hat{\theta}=\left\{\hat{\theta}_{\mathbf{k}}\right\}_{\mathbf{k} \in \overline{\mathbb{Z}}^{2}}$ such that

$$
\overline{\hat{\theta}}_{-\mathbf{k}}=-\hat{\theta}_{\mathbf{k}}
$$

(reality condition), and that for any positive integer $m$

$$
\sup _{\mathbf{k} \in \overline{\mathbb{Z}}^{2}} k^{m}\left|\hat{\theta}_{\mathbf{k}}\right|<\infty
$$

(condition of rapid decrease).

By setting

$$
\|\hat{\theta}\|_{n} \equiv\left(\sum_{\mathbf{k} \in \overline{\mathbb{Z}}^{2}} k^{2 n}\left|\hat{\theta}_{\mathbf{k}}\right|^{2}\right)^{1 / 2}
$$

we have $\|\hat{\theta}\|_{n}=\|\underline{\theta}\|_{n}$. We denote by $\tilde{s}$ the linear space of the sequences satisfying conditions (3.1) and (3.2). Definition (3.3) gives us a family of seminorms on $\tilde{s}$ and it is easily seen that the following proposition holds:

Proposition 3.1. The map $\underline{\theta} \rightarrow \hat{\theta}$ (Fourier transform) of $\tilde{\mathscr{S}}\left(T^{2}\right)$ on $\tilde{s}$, endowed with the topology generated by the seminorms (3.3), is a topological isomorphism. 
Consider the space $\tilde{\mathscr{S}}^{\prime}\left(T^{2}\right)$, dual to $\tilde{\mathscr{S}}\left(T^{2}\right)$. We shall denote by $\langle\mathbf{u}, \underline{\theta}\rangle$ the action of $\mathbf{u} \in \tilde{\mathscr{S}}^{\prime}\left(T^{2}\right)$ on $\underline{\theta} \in \tilde{\mathscr{S}}\left(T^{2}\right)$. The Fourier transform on $\tilde{\mathscr{S}}^{\prime}\left(T^{2}\right)$ is the map $\mathbf{u} \rightarrow \hat{u} \equiv\left\{\hat{u}_{\mathbf{k}}\right\}_{\mathbf{k} \in \overline{\mathbb{Z}}^{2}}$ defined by setting

$$
\hat{u}_{\mathbf{k}}=\overline{\left\langle\mathbf{u}, \frac{\mathbf{k}^{\perp}}{k 2 \pi} \exp (i \mathbf{k} \cdot \mathbf{x})\right\rangle}
$$

Since $\mathbf{u} \in \tilde{\mathscr{S}}^{\prime}\left(T^{2}\right)$, the sequence $\left\{\hat{u}_{\mathbf{k}}\right\}_{\mathbf{k} \in \overline{\mathbb{Z}}^{2}}$ satisfies the reality condition

$$
\overline{\hat{u}}_{-\mathbf{k}}=-\hat{u}_{-\mathbf{k}}
$$

and is low increase, i.e. there exist positive constants $c$ and $N$ depending on $\mathbf{u}$ such that

$$
\left|\hat{u}_{\mathbf{k}}\right|<c k^{N} \text {. }
$$

We denote by $\tilde{s}^{\prime}$ the space of sequences satisfying conditions $\left(3.1^{\prime}\right)$ and $\left(3.2^{\prime}\right) . \tilde{s}^{\prime}$ is the dual of $\tilde{s}$ according to the duality

$$
\langle\hat{u}, \hat{\theta}\rangle=\sum_{\mathbf{k} \in \overline{\mathbb{Z}}^{2}} \overline{\hat{u}}_{\mathbf{k}} \hat{\theta}_{\mathbf{k}} \quad\left(\hat{u} \in \tilde{S}^{\prime}, \hat{\theta} \in \tilde{S}\right) .
$$

The following proposition is easily seen to be true:

Proposition 3.2. The map $\mathbf{u} \rightarrow \hat{u}$ (Fourier transform) of $\tilde{\mathscr{S}}^{\prime}\left(T^{2}\right)$ on $\tilde{s}^{\prime}$, endowed with the weak topology corresponding to the duality (3.4), is a topological isomorphism.

$\tilde{s}^{\prime}$ is a nice space to place measures because it is the dual of a nuclear space, and therefore any continuous cylindric Borel measure on it is $\sigma$-continuous (cf. [13], Chap. 4). Moreover any such measure corresponds to a generalized random field (hereafter g.r.f.) (cf. [13], Chap. 3) on $T^{2}$, so that we can make use of the general results of the theory of g.r.f.'s.

Before coming to the evolution of measures on $\tilde{s}^{\prime}$ corresponding to the full Euler equation, we define the evolution associated to f.d.a.'s (Definition 2.1).

Definition 3.1. Consider the index sets $I_{N}=\left\{\mathbf{k} \in \overline{\mathbb{Z}}^{2}|| \mathbf{k} \mid \leqq N\right\},(N=1,2, \ldots)$. For any $N$ we define an evolution group in $\tilde{s}^{\prime}, T_{t}^{(N)}$, by setting:

$$
\left(T_{t}^{(N)} \hat{u}\right)_{\mathbf{k}}= \begin{cases}u_{\mathbf{k}}^{(N)}(t) & \mathbf{k} \in I_{N} \\ \hat{u}_{\mathbf{k}} & \mathbf{k} \notin I_{N}\end{cases}
$$

$u_{\mathbf{k}}^{(N)}(t)$ being the solution of the f.d.a. corresponding to the index set $I_{N}$ (Definition 2.1) with initial data $u_{\mathbf{k}}^{(N)}(0)=\hat{u}_{\mathbf{k}}, \mathbf{k} \in I_{N}$. define

Suppose $\mu_{0}$ is a cylindric Borel measure on $\tilde{s}^{\prime}$. For any measurable set $A \subset \tilde{s}^{\prime}$

$$
\mu_{t}^{(N)}(A)=\mu_{0}\left(T_{-t}^{(N)} A\right) .
$$

We get a family of measures $\left\{\mu_{t}^{(N)}\right\}_{t \in \mathbb{R}^{1}}$ for any $N$.

Let $\left\{\Phi_{t}^{(N)}(\cdot)\right\}_{t \in \mathbb{R}^{1}}$, be the corresponding family of characteristic functionals defined on $\tilde{s}$ :

$$
\Phi_{t}^{(N)}(\hat{\theta})=\int_{\tilde{s}^{\prime}} \exp (i\langle\hat{u}, \hat{\theta}\rangle) d \mu_{t}^{(N)}(\hat{u})
$$


Proposition 3.3. If $\int_{\tilde{s}^{\prime}}\left|\hat{u}_{\mathbf{k}}\right|^{2} d \mu_{0}<\infty, \forall \mathbf{k} \in \overline{\mathbb{Z}}^{2}, \Phi_{t}^{(N)}$ is differentiable with respect to $t$ for any $N$ and satisfies the equation

$$
\frac{\partial}{\partial t} \Phi_{t}^{(N)}(\hat{\theta})=i \int_{\tilde{s}^{\prime}}\left\langle B^{(N)}(\hat{u}), \hat{\theta}\right\rangle \exp (i\langle\hat{u}, \hat{\theta}\rangle) d \mu_{t}^{(N)}(\hat{u}),
$$

where $\mathbf{B}^{(N)}(\hat{u}) \in \tilde{S}^{\prime}$ is the vector with components

$$
\begin{array}{ll}
\mathbf{B}_{\mathbf{k}}^{(N)}(\hat{u})=-i \sum_{\substack{\mathbf{h}+\mathbf{m}=\mathbf{k} \\
\mathbf{h}, \mathbf{m} \in I_{N}}} \Gamma_{\mathbf{h m k}} \hat{u}_{\mathbf{h}} \hat{u}_{\mathbf{m}} & \left(\mathbf{k} \in I_{N}\right) \\
\mathbf{B}_{\mathbf{k}}^{(N)}(\hat{u})=0 & \left(\mathbf{k} \notin I_{N}\right) .
\end{array}
$$

Proof. The proof consists in a change of variables and an application of the Lebesgue dominated convergence theorem to ensure derivability under the integral sign.

We could now introduce the Hopf equation by passing (formally) to the limit $N \rightarrow \infty$. However the limit of $B^{(N)}(\hat{u})$ is unfortunately not defined everywhere in $\tilde{s}^{\prime}$, so that we must formulate some additional assumptions on the measures. We first derive the following simple:

Proposition 3.4. Suppose we have a sequence of functions $F^{(N)}: \tilde{s}^{\prime} \rightarrow \tilde{S}^{\prime}$ and a $\sigma$ additive measure $\mu$ on $\tilde{s}^{\prime}$, such that $\left\langle F^{(N)}(\hat{u}), \hat{\theta}\right\rangle$ is a converging sequence in $L^{1}(d \mu)$ for any $\hat{\theta} \in \tilde{s}$. Then there exist a function $F: \tilde{s}^{\prime} \rightarrow \tilde{s}^{\prime}$, and a subsequence $F^{\left(N_{i}\right)}$ such that $F^{\left(N_{i}\right)}(\hat{u}) \underset{N \rightarrow \infty}{\longrightarrow} F(\hat{u})$ in $\tilde{s}^{\prime}$ almost everywhere with respect to $\mu$ (hereafter $\mu$-a.e.), and

$$
\left\langle F^{(N)}(\hat{u}), \hat{\theta}\right\rangle \stackrel{L^{1}(d \mu)}{\longrightarrow}\langle F(\hat{u}), \hat{\theta}\rangle .
$$

Proof. In fact, since the sequence $\int_{\tilde{s}^{\prime}}\left|\left\langle F^{(N)}(\hat{u}), \hat{\theta}\right\rangle\right| d \mu(\hat{u})$ converges, it is limited for any $\hat{\theta} \in \tilde{S}$. Therefore (cf. [14], Theorem V.7) there exist two constants $m$ and $c$ such that:

$$
\int_{\tilde{s}^{\prime}}\left|\left\langle F^{(N)}(\hat{u}), \hat{\theta}\right\rangle\right| d \mu(\hat{u}) \leqq c\|\hat{\theta}\|_{m}
$$

for all $N$. Since $\left\langle F^{(N)}(\hat{u}), \hat{\theta}\right\rangle$ converges in $L^{1}(d \mu)$ for any fixed $\hat{\theta} \in \tilde{S}$, the Fourier components $F_{\mathbf{k}}^{(N)}$ converge in $L^{1}(d \mu)$ for any fixed $\mathbf{k}$. Denoting by $F_{\mathbf{k}}(\hat{u})$ the corresponding limits we have for any $\backslash \mathbf{k} \in \overline{\mathbb{Z}}^{2}$

$$
\int_{\tilde{s}^{\prime}} \frac{\left|F_{\mathbf{k}}(\hat{u})\right|}{k^{m}} d \mu(\hat{u}) \leqq c .
$$

Because of $L^{1}$ convergence $F_{\mathbf{k}}(\hat{u})$ is $\mu$-a.e. the limit of a subsequence $F_{\mathbf{k}}^{\left(N_{\imath}\right)}(\hat{u})$. By a diagonal procedure it is possible to find a subsequence $F_{\mathbf{k}}^{\left(N_{j}\right)} \mu$-a.e. converging to $F_{\mathbf{k}}$, for all $\mathbf{k} \in \overline{\mathbb{Z}}^{2}$. It is easily seen that the sequence $F_{\mathbf{k}}(\hat{u}) / k^{m+n}$ converges to $0 \mu$-a.e. as $|\mathbf{k}| \rightarrow \infty$ for $n>2$, so that $\mu$-a.e. there exist a fixed integer $m^{\prime}$, and a constant depending on $\hat{u}$ such that

$$
\left|F_{\mathbf{k}}(\hat{u})\right|<c(\hat{u}) k^{m^{\prime}},
$$

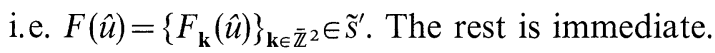


Definition 3.2. We shall say that the family of cylindric Borel measures on $\tilde{s}^{\prime}$ $\left\{\mu_{t}\right\}_{t \in[0, T]}$ is a solution of the Hopf equation associated to the incompressible Euler equation on $T^{2}$ with the initial condition $\mu^{(0)}$, whenever the characteristic functional $\Phi_{t}(\hat{\theta})=\int_{\tilde{s}^{\prime}} \exp (i\langle\hat{u}, \hat{\theta}\rangle) d \mu_{t}(\hat{u})$ is derivable almost everywhere with respect to $t, \Phi_{0}(\hat{\theta})=\Phi^{(0)}(\hat{\theta})=\int_{\tilde{s}^{\prime}} \exp (i\langle\hat{u}, \phi\rangle) d \mu^{(0)}(\hat{u})$, and the following equality takes place almost everywhere with respect to the Lebesgue measure on $[0, T]$

$$
\frac{\partial}{\partial t} \Phi_{t}(\hat{\theta})=i \int_{\tilde{s}^{\prime}}\langle B(\hat{u}), \hat{\theta}\rangle \exp (i\langle\hat{u}, \hat{\theta}\rangle) d \mu_{t}(\hat{u})
$$

$B(\hat{u})$ being the $L^{1}\left(d \mu_{t}\right)$ - limit of $B^{(N)}(\hat{u})$, defined by Eq. (3.5).

In the following we shall be interested only in the stationary equation

$$
\int_{\tilde{s}^{\prime}}\langle B(\hat{u}), \hat{\theta}\rangle \exp (i\langle\hat{u}, \hat{\theta}\rangle) d \mu(\hat{u})=0 .
$$

Definition 3.3. We shall call "equilibrium state" any cylindric Borel measure on $\tilde{s}^{\prime}$ satisfying Eq. (3.7).

\section{Equilibrium States Associated to the Vortex Conservation Law}

As we said in Sect. 2 a characteristic feature of the two-dimensional Euler fluid is the conservation law of vorticity for fluid particles (2.8).

Since the "density of fluid particles" (i.e. volumes) is preserved, because of the solenoidality condition (2.2), we are led to the conclusion that vorticity distributions which are independent at each point should correspond to equilibrium states.

According to a well known result (cf. [13], Chap. III) a general class of real generalized random fields with independent values at each point is identified by the class of characteristic functionals

$$
\chi_{\psi}(\phi)=\exp \int_{T^{2}} \psi(\phi(\mathbf{x})) d x \quad\left(\psi \in \mathscr{I}, \phi \in \mathscr{S}\left(T^{2}\right)\right) .
$$

( $\mathscr{I}$ is the class of complex functions which are logarithms of the characteristic functions of an infinitely divisible distribution law.)

We shall presently show that the measures associated to the characteristic functionals (4.1) are limits of natural "statistical mechanical" lattice measures with "Gibbs factor" associated to the constants of the motion $I_{f}$ defined by (2.10).

A statistical mechanical measure with "Gibbs factor" associated to $I_{f}$ may be written formally as

$$
d \mu_{f}(\mathbf{u})=M^{-1} \exp \left(\int_{T^{2}} f(\operatorname{rot} \mathbf{u}(\mathbf{x})) d x\right)^{\prime \prime} \prod_{\mathbf{x} \in T^{2}} d(\operatorname{rot} \mathbf{u}(\mathbf{x}))^{\prime \prime}
$$

(where $M$ is some normalization constant).

Consider the lattice $\mathbb{Z}_{N} \subset \mathbb{Z}^{2}$

$$
\mathbb{Z}_{N}=\left\{\mathbf{k} \in \mathbb{Z}^{2} \mid \mathbf{k} / N \in[0,2 \pi) \times[0,2 \pi)\right\} \quad N=1,2, \ldots
$$


set $n_{N}=$ card $\left\{\mathbb{Z}_{N}\right\}$, denote $\xi_{\mathbf{k}}^{(N)}$ the vorticity of the point $\mathbf{k} / N$, and by $\xi^{(N)}$ the $n_{N^{-}}$ dimensional vector $\left\{\xi_{\mathbf{k}}^{(N)}\right\}_{\mathbf{k} \in \mathbb{Z}_{N}}$. The measure

$$
d \mu_{N}\left(\xi^{(N)}\right)=\prod_{\mathbf{k} \in \mathbb{Z}_{N}} \varrho_{N}\left(\xi_{\mathbf{k}}^{(N)}\right) d \xi_{\mathbf{k}}^{(N)}
$$

with $d \xi_{\mathbf{k}}^{(N)}$ the Lebesgue measure on $\mathbb{R}^{1}, \varrho_{N}$ a nonnegative function such that $\left.\int_{\infty}^{\infty} \varrho_{N}(x) d x=1\right]$ is a natural lattice approximation of the meanigless expression (4.2).

For any $\phi \in \mathscr{S}\left(T^{2}\right)$ consider the random variable

$$
\xi^{(N)}(\phi)=\sum_{\mathbf{k} \in \mathbb{Z}_{N}} \phi(\mathbf{k} / N) \xi_{\mathbf{k}}^{(N)}
$$

the collection of all such random variables for all $\phi \in \mathscr{S}\left(T^{2}\right)$ gives us a random linear functional, that is a g.r.f. on $T^{2}$ which we shall denote by $\xi^{(N)}(\cdot)$.

Definition 4.1. We shall say that g.r.f.'s $\xi^{(N)}(\cdot)$ converge weakly as $N \rightarrow \infty$ to the g.r.f. $\xi(\cdot)$ whenever the joint distribution functions of the random variables $\xi^{(N)}\left(\phi_{1}\right) \ldots \xi^{(N)}\left(\phi_{n}\right)$ converge weakly to the joint distribution functions of $\xi\left(\phi_{1}\right) \ldots \xi\left(\phi_{n}\right)$, for any choice of $\phi_{1}, \ldots, \phi_{n} \in \mathscr{S}\left(T^{2}\right)$ and of the positive integer $n$.

The classical theorems on the convergence of sums of independent random variables allow us to find out the class of all possible weak limits of the g.r.f.'s $\xi^{(N)}(\cdot)$, under the usual natural restriction that the contribution at each single term in the sum (4.3) is, for large $N$, infinitesimally small. It is easily seen that this is ensured by the following "infinitesimality condition": for all $\varepsilon>0$ we should have

$$
\lim _{N \rightarrow \infty} \int_{|x|>\varepsilon} \varrho_{N}(x) d x=0
$$

Theorem 4.1. Let $a_{\tau}^{(N)}=\int_{|x|<\tau} x \varrho_{N}(x) d x(\tau>0)$. A necessary and sufficient condition in order that the sequence of g.r.f.'s $\xi^{(N)}$ subject to conditions (4.4), converge weakly to a limit, is that the functions

$$
\psi_{N}(t)=N^{2} \int_{-\infty}^{\infty}(\exp (i t x)-1) \varrho_{N}\left(x+a_{\tau}^{(N)}\right) d x
$$

converge everywhere to a continuous limit. The set of all possible weak limits is the set of g.r.f.'s the characteristic functionals of which are given by

$$
\chi_{\psi}(\phi)=\exp \int_{T^{2}} \psi(\phi(x)) d x \quad \phi \in \mathscr{S}\left(T^{2}\right)
$$

where $\psi \in \mathscr{I}$ and has Levy-Khinchin representation of the type

$$
\psi(t)=\int_{-\infty}^{\infty}\left(\exp (i t x)-1-\frac{i t x}{1+x^{2}}\right) \frac{1+x^{2}}{x^{2}} d F(x)
$$

$F(x)$ being a nondecreasing limited function such that $F(-\infty)=0$.

Proof. First of all note that, because of linearity, weak convergence of the g.r.f.'s $\xi^{(N)}$ is equivalent to weak convergence of the distribution functions of the random 
variables $\xi^{(N)}(\phi)$ for any fixed $\phi \in \mathscr{S}\left(T^{2}\right)$ [in fact the characteristic function of the joint distribution of the random variables $\left\{\xi^{(N)}\left(\phi_{1}\right), \ldots, \xi^{(N)}\left(\phi_{N}\right)\right\}$ calculated at the point $\left(t_{1}, \ldots, t_{n}\right)$ is just the characteristic function of $\xi^{(N)}\left(t_{1} \phi_{1}+\ldots+t_{n} \phi_{n}\right)$ calculated at $t=1]$. Now the characteristic function of $\xi^{(N)}(\phi)$ is given by

$$
G_{\phi}^{(N)}(t)=\prod_{\mathbf{k} \in \mathbb{Z}_{N}} f_{N}(t \phi(\mathbf{k} / N))
$$

where $f_{N}(t)=\int_{-\infty}^{\infty} \exp (i t x) \varrho_{N}(x) d x$. Because of condition (4.4) the functions $g_{\phi}^{(N)}(t)=\log G_{\phi}^{(N)}(t)$ are finite in any finite interval for $N$ large enough, and for any $\tau>0$ we have

$$
\begin{aligned}
g_{\phi}^{(N)}(t)= & i t a_{\tau}^{(N)} \sum_{\mathbf{k} \in \mathbb{Z}_{N}} \phi(\mathbf{k} / N) \\
& +\sum_{\mathbf{k} \in \mathbb{Z}_{N}} \log \left\{\int_{-\infty}^{\infty} \exp (i t \phi(\mathbf{k} / N)) \varrho_{N}\left(x+a_{\tau}^{(N)}\right) d x\right\} .
\end{aligned}
$$

Since $a_{\tau}^{(N)} \underset{N \rightarrow \infty}{\longrightarrow} 0$ in force of condition (4.4), and since $\int_{T^{2}} \phi(x) d x=0$ we have

$$
a_{\tau}^{(N)} \sum_{\mathbf{k} \in \mathbb{Z}_{N}} \phi(\mathbf{k} / N) \underset{N \rightarrow \infty}{\longrightarrow} 0 .
$$

Moreover by repeating the classical argument of Kolomogorov and Gnedenko [15] with minor modifications, it is readily seen that pointwise convergence of the sequence $\left\{g_{\phi}^{(N)}(t)\right\}_{N=1}^{\infty}$ is equivalent to pointwise convergence of the following sequence

$$
\tilde{g}_{\phi}^{(N)}(t)=\sum_{\mathbf{k} \in \mathbb{Z}_{N}} \int_{-\infty}^{\infty}(\exp (i t \phi(\mathbf{k} / N))-1) \varrho_{N}\left(x+a_{\tau}^{(N)}\right) d x
$$

and that $\lim _{N \rightarrow \infty} \tilde{g}_{\phi}^{(N)}(t)=\lim _{N \rightarrow \infty} g_{\phi}^{(N)}(t)$. Now $\tilde{g}_{\phi}^{(N)}(t)$ is nothing else than a Riemann sum of the integral $\int_{T^{2}} \psi_{N}(\phi(x)) d x$, and the first assertion of the theorem follows easily by noting that we are dealing with functions for which pointwise convergence is equivalent to uniform convergence on any compact set. The second assertion follows from the fact that the limit law of a sum of independent variables subject to the infinitesimality condition is necessarily an infinitely divisible law and from the observation that if in the Levy-Khinchin representation of $\psi$

$$
\psi(t)=i \gamma t+\int_{-\infty}^{\infty}\left(\exp (i t x)-1-\frac{i t x}{1+x^{2}}\right) d F(x)
$$

we change the value of $\gamma$ the characteristic functional (4.5) does not change [because of the condition $\int_{T^{2}} \phi(x) d x=0$ ].

Once we have the characteristic functional $\chi$ of the vorticity distribution we obtain the corresponding characteristic functional of the velocity distribution $\Phi$ by setting

$$
\Phi(\operatorname{rot} \phi)=\chi(\phi) .
$$


So that the characteristic functional of the velocity distribution corresponding to the functional (4.5) is

$$
\Phi_{\psi}(\underline{\theta})=\exp \int_{T^{2}} \psi\left(G(\operatorname{rot} \underline{\theta})(x) d x \quad\left(\underline{\theta} \in \tilde{\mathscr{S}}\left(T^{2}\right)\right) .\right.
$$

Any g.r.f. corresponding to such a characteristic functional possesses a realization on $\tilde{\mathscr{S}}^{\prime}\left(T^{2}\right)$ given by a cylindric Borel measure (which in uniquely determined), and therefore, by Proposition 3.2, a realization on $\tilde{s}^{\prime}$ with the same properties (cf. [13], Chap. IV). The characteristic functional of the corresponding measure on $\tilde{s}^{\prime}$ is obtained simply by substituting for $\underline{\theta}$ its Fourier development in (4.6) :

$$
\Phi_{\psi}(\hat{\theta})=\exp \int_{T^{2}} \psi\left(\sum_{\mathbf{k} \in \overline{\mathbb{Z}}^{2}} \frac{i}{k} \hat{\theta}_{\mathbf{k}} e^{i \mathbf{k} \cdot \mathbf{x}}\right) d x .
$$

Theorem 4.2. If $\psi(\cdot)$ is the logarithm of an infinitely divisible distribution law with $\psi^{\mathrm{IV}}(0)<\infty$, the measure $\mu_{\psi}$ on $\tilde{s}^{\prime}$ identified by the characteristic functional (4.7) is a solution of the stationary Hopf equation (3.7).

Proof. First of all we show that the sequence $\left\langle B^{(N)}(\hat{u}), \hat{\theta}\right\rangle$ converges in $L^{2}\left(d \mu_{\psi}\right)$ as $N \rightarrow \infty$. Setting $Z^{(N)}(\hat{u})=B^{(N+1)}(\hat{u})-B^{(N)}(\hat{u})$ and denoting by $\mathscr{E}_{\psi}\{\cdot\}$ the average with respect to $\mu_{\psi}$ we have (we write for short $\Gamma_{\mathbf{m}, \mathbf{k}}$ in place of $\Gamma_{\mathbf{m}, \mathbf{k}-\mathbf{m}, \mathbf{k}}$ )

where

$$
\begin{aligned}
& \left\|\left\langle Z^{(N)}(\hat{u}), \hat{\theta}\right\rangle\right\|_{L^{2}\left(d \mu_{\psi}\right)}^{2}=\sum_{\mathbf{k}, \mathbf{h} \in I_{N+1}} \overline{\hat{\theta}}_{\mathbf{k}} \hat{\theta}_{\mathbf{h}} \mathscr{E}_{\psi}\left\{Z_{\mathbf{k}}^{(N)} \overline{Z_{\mathbf{h}}^{(N)}}\right\} \\
& =\sum_{\mathbf{k}, \mathbf{h} \in I_{N}} \overline{\hat{\theta}}_{\mathbf{k}} \hat{\theta}_{\mathbf{h}} \sum_{\substack{\mathbf{m} \in H_{\mathbf{k}}^{(N)} \\
\mathbf{m}^{\prime} \in H_{\mathbf{h}}^{(N)}}} R_{\mathbf{m}, \mathbf{m}^{\prime}}^{\mathbf{k}, \mathbf{h}}+2 \sum_{\substack{\mathbf{k} \in I_{N}+1 \backslash I_{N} \\
\mathbf{h} \in I_{N}}} \overline{\hat{\theta}}_{\mathbf{k}} \hat{\theta}_{\mathbf{h}} \sum_{\substack{\mathbf{m} \\
\mathbf{k} \in \mathbf{m}, H_{\mathbf{h}}^{(N)}}} R_{\mathbf{m}, \mathbf{m}^{\prime},+}^{\mathbf{k}, \mathbf{h}} \\
& +\sum_{\mathbf{k}, \mathbf{h} \in I_{N+1} \backslash I_{N}} \overline{\hat{\theta}}_{\mathbf{k}} \hat{\theta}_{\mathbf{h}} \sum_{\substack{\mathbf{k}-\mathbf{m}, \mathbf{m} \in I_{N}+1 \\
\mathbf{h}-\mathbf{m}^{\prime}, \mathbf{m}^{\prime} \in I_{N}+1}} R_{\mathbf{m}, \mathbf{m}^{\prime}}^{\mathbf{k}, \mathbf{h}} \quad\left(R_{\mathbf{m}, \mathbf{m}^{\prime}}^{\mathbf{k}, \mathbf{h}}=\Gamma_{\mathbf{m}, \mathbf{k}} \Gamma_{\mathbf{m}^{\prime}, \mathbf{h}} \mathscr{E}_{\psi}\left\{\hat{u}_{\mathbf{m}} \hat{u}_{\mathbf{k}-\mathbf{m}} \overline{\hat{u}}_{\mathbf{m}^{\prime}} \overline{\hat{u}}_{\mathbf{h}-\mathbf{m}}\right\}\right)
\end{aligned}
$$

$$
\begin{aligned}
H_{\mathbf{k}}^{(N)}= & \left\{\mathbf{m} \in \overline{\mathbb{Z}}^{2} \mid \mathbf{m} \in I_{N+1} \backslash I_{N}, \mathbf{k}-\mathbf{m} \in I_{N+1} \backslash I_{N}\right\} \cup\left\{\mathbf{m} \in \overline{\mathbb{Z}}^{2} \mid \mathbf{m} \in I_{N+1} \backslash I_{N}, \mathbf{k}-\mathbf{m} \in I_{N}\right\} \\
& \cup\left\{\mathbf{m} \in \overline{\mathbb{Z}}^{2} \mid \mathbf{m} \in I_{N}, \mathbf{k}-\mathbf{m} \in I_{N+1} \backslash I_{N}\right\} .
\end{aligned}
$$

Since

$$
\begin{aligned}
\mathscr{E}_{\psi}\left\{\hat{u}_{\mathbf{k}} \hat{u}_{\mathbf{h}} \hat{u}_{\mathbf{m}} \hat{u}_{\mathbf{n}}\right\} & =\frac{1}{k h m n}\left\{\left(\psi^{\prime \prime}(0)\right)^{2}[\delta(\mathbf{k}+\mathbf{h}) \delta(\mathbf{m}+\mathbf{n})+\delta(\mathbf{k}+\mathbf{m}) \delta(\mathbf{h}+\mathbf{m})\right. \\
& \left.+\delta(\mathbf{k}+\mathbf{n}) \delta(\mathbf{h}+\mathbf{m})]+\psi^{\mathrm{IV}}(0) \delta(\mathbf{k}+\mathbf{h}+\mathbf{m}+\mathbf{n})\right\}
\end{aligned}
$$

$\left[\delta(\mathbf{k})\right.$ is the Kronecker symbol: $\delta(\mathbf{k})=\left\{\begin{array}{lll}1 & \text { for } & k=0 \\ 0 & \text { for } & k \neq 0\end{array}\right]$, by substitution we get

$$
\begin{aligned}
\left\|\left\langle Z^{(N)}(\hat{u}), \hat{\theta}\right\rangle\right\|_{L^{2}\left(d \mu_{\psi}\right)}^{2}= & \sum_{\mathbf{k} \in I_{N}}\left|\hat{\theta}_{\mathbf{k}}\right|^{2}\left\{2\left(\psi^{\prime \prime}(0)\right)^{2} \sum_{\mathbf{m} \in \boldsymbol{H}_{\mathbf{k}}^{(N)}} \frac{\Gamma_{\mathbf{m}, \mathbf{k}}^{2}}{m^{2}|\mathbf{k}-\mathbf{m}|^{2}}\right. \\
& \left.+\psi^{\mathrm{IV}}(0)\left(\sum_{\mathbf{m} \in H_{\mathbf{k}}^{(N)}} \frac{\Gamma_{\mathbf{m}, \mathbf{k}}}{m|\mathbf{k}-\mathbf{m}|}\right)^{2}\right\} \\
+ & \sum_{\mathbf{k} \in I_{N+1} \backslash I_{N}}\left|\hat{\theta}_{\mathbf{k}}\right|^{2}\left\{2\left(\psi^{\prime \prime}(0)\right)^{2} \sum_{\mathbf{m} \in I_{N+1}} \frac{\Gamma_{\mathbf{m}, \mathbf{k}}^{2}}{m^{2}|\mathbf{k}-\mathbf{m}|^{2}}+\psi^{\mathrm{IV}}(0)\left(\sum_{\mathbf{m} \in I_{N+1}} \frac{\Gamma_{\mathbf{m}, \mathbf{k}}}{m|\mathbf{k}-\mathbf{m}|}\right)^{2}\right\} .
\end{aligned}
$$


Now observe that if $\mathbf{m} \in H_{\mathbf{k}}^{(N)}\left(I_{N+1}\right)$ so does $\tilde{\mathbf{m}}=\mathbf{m}-2 \frac{\left(\mathbf{m} \cdot \mathbf{k}^{\perp}\right)}{k^{2}} \mathbf{k}^{\perp}$ (because $|\tilde{\mathbf{m}}|=|\mathbf{m}|$ and $|\mathbf{k}-\tilde{\mathbf{m}}|=|\mathbf{k}-\mathbf{m}|)$ and it is easily seen that:

$$
\Gamma_{\mathbf{m}, \mathbf{k}}=-\Gamma_{\tilde{\mathbf{m}} \mathbf{k}},
$$

so that

$$
\sum_{\mathbf{m} \in H_{(\mathbf{k}}^{(N)}} \frac{\Gamma_{\mathbf{m}, \mathbf{k}}}{m|\mathbf{k}-\mathbf{m}|}=\sum_{\mathbf{m} \in I_{N}+1} \frac{\Gamma_{\mathbf{m}, \mathbf{k}}}{m|\mathbf{k}-\mathbf{m}|}=0 .
$$

Since moreover

$$
\bigcup_{n=1}^{N} H_{\mathbf{k}}^{(n)}=\left\{\mathbf{m} \in \overline{\mathbb{Z}}^{2} \mid \mathbf{m}, \mathbf{k}-\mathbf{m} \in I_{N+1}\right\}
$$

we have:

$$
\sum_{n=1}^{N}\left\|\left\langle Z^{(n)}(\hat{u}), \hat{\theta}\right\rangle\right\|_{L^{2}\left(d \mu_{\psi}\right)}^{2}=\frac{1}{2}\left(\psi^{\prime \prime}(0)\right)^{2} \sum_{\mathbf{k} \in I_{N+1}}\left|\hat{\theta}_{\mathbf{k}}\right|^{2} \sum_{\substack{\mathbf{m} \in I_{N}^{+1} \\ \mathbf{k}-\mathbf{m} \in I_{N+1}}} \frac{\left(\mathbf{m}^{\perp} \cdot \mathbf{k}\right)^{2}\left(k^{2}-\mathbf{m} \cdot \mathbf{k}\right)^{2}}{k^{2} m^{4}|\mathbf{k}-\mathbf{m}|^{4}}
$$

and, in addition, the series $\sum_{n=1}^{\infty}\left\|\left\langle Z^{(n)}(\hat{u}), \hat{\theta}\right\rangle\right\|_{L^{2}\left(d \mu_{\psi}\right)}$ converges.

Since

$$
\left\langle B_{\mathbf{k}}^{(N)}(\hat{u}), \hat{\theta}\right\rangle=\sum_{n=1}^{N-1}\left\langle Z^{(n)}(\hat{u}), \hat{\theta}\right\rangle,
$$

by applying the theorem of Beppo Levi and the dominated convergence theorem, it follows that the sequence $\left\langle B^{(N)}(\hat{u}), \hat{\theta}\right\rangle$ converges in $L^{2}\left(d \mu_{\psi}\right)$ as well as $\mu_{\psi}$-a.e. Therefore the left hand side of the stationary Hopf equation (3.7) makes sense, the function $B(\hat{u})$ which appears in it being the limit, in the sense of Proposition 3.4, of the functions $B^{(N)}(\hat{u})$, as $N \rightarrow \infty$. [Notice that we have shown that the sequence $B^{(N)}(\hat{u})$ itself converges $\mu_{\psi}$-a.e.] We have

$$
\begin{aligned}
& \int_{\tilde{s}^{\prime}}\langle B(\hat{u}), \hat{\theta}\rangle \exp (i\langle\hat{u}, \hat{\theta}\rangle) d \mu(\hat{u})=\lim _{N \rightarrow \infty} \int_{\tilde{s}^{\prime}}\left\langle B^{(N)}(\hat{u}), \hat{\theta}\right\rangle \exp (i\langle\hat{u}, \hat{\theta}\rangle) d \mu(\hat{u}) \\
& =\lim _{N \rightarrow \infty}-\sum_{\mathbf{k} \in I_{N}} \hat{\theta}_{\mathbf{k}}\left\{\sum_{\mathbf{k}-\mathbf{m}, \mathbf{m} \in I_{N}} \Gamma_{\mathbf{m}, \mathbf{k}} \int_{T^{2}} \psi^{\prime}(\sigma(\mathbf{x})) \exp (i \mathbf{m} \cdot \mathbf{x}) / m d x\right. \\
& \cdot \int_{T^{2}} \psi^{\prime}(\sigma(\mathbf{x})) \exp (i(\mathbf{k}-\mathbf{m}) \cdot \mathbf{x}) /|\mathbf{k}-\mathbf{m}| d x+\int_{T^{2}} \psi^{\prime \prime}(\sigma(\mathbf{x})) \exp (i \mathbf{k} \cdot \mathbf{x}) d x \\
& \left.\cdot\left(\sum_{\mathbf{k}-\mathbf{m}, \mathbf{m} \in I_{N}} \frac{\Gamma_{\mathbf{m}, \mathbf{k}}}{m|\mathbf{k}-\mathbf{m}|}\right)\right\} \quad\left[\text { where } \sigma(\mathbf{x})=\sum_{\mathbf{k} \in \overline{\mathbb{Z}}^{2}} \frac{i}{k} \hat{\theta}_{\mathbf{k}} \exp (i \mathbf{k} \cdot \mathbf{x})\right] .
\end{aligned}
$$

Using (4.8) we see that the second term in the curly brackets gives no contribution, and since the remaining double series converges absolutely, we have

$$
\begin{gathered}
\int_{s^{\prime}}\langle B(\hat{u}), \theta\rangle \exp (i\langle\hat{u}, \hat{\theta}\rangle) d \mu(\hat{u})=i \sum_{\mathbf{k} \in \overline{\mathbb{Z}}^{2}} \sigma_{\mathbf{k}}\left\{\sum_{\mathbf{k}-\mathbf{m}, \mathbf{m} \in \overline{\mathbb{Z}}^{2}} \frac{\mathbf{m}^{\perp} \cdot \mathbf{k}}{m^{2}} \int_{T^{2}} \psi^{\prime}(\sigma(\mathbf{x})) \exp (i \mathbf{m} \cdot \mathbf{x}) d x\right\} \\
\cdot \int_{T^{2}} \psi^{\prime}(\sigma(\mathbf{x})) \exp (i(\mathbf{k}-\mathbf{m}) \cdot \mathbf{x}) d x=i \int_{T^{2}} \nabla \psi(\sigma(\mathbf{x})) \cdot \operatorname{rot} G\left(\psi^{\prime}(\sigma(\cdot))\right) d x=0 .
\end{gathered}
$$




\section{Properties of the Gaussian and Poissonian States}

a. Gaussian States

For $\psi(t)=\frac{-1}{2} \sigma^{2} t^{2}(\sigma>0)$ we have a gaussian equilibrium state. In fact its characteristic functional is :

$$
\Phi_{\psi}(\hat{\theta})=\exp \left(\frac{-1}{2} \sigma^{2} \sum_{\mathbf{k} \in \overline{\mathbb{Z}}^{2}} \frac{\left|\hat{\theta}_{\mathbf{k}}\right|^{2}}{k^{2}}\right) .
$$

Denoting by $\mu_{\sigma}$ the corresponding measure on $\tilde{s}^{\prime}$, the measure of any cylindric set $A$ is given by

$$
\mu_{\sigma}(A)=\int_{A} \prod_{\mathbf{k} \in I} \exp \left(-k^{2}\left|\hat{u}_{\mathbf{k}}\right|^{2} / 2 \sigma^{2}\right) \delta\left(\bar{u}_{-\mathbf{k}}+\hat{u}_{\mathbf{k}}\right) d^{2} \hat{u}_{\mathbf{k}} /\left(\pi^{1 / 2} \sigma / k\right)
$$

(here $I$ is the set of integers corresponding to the variables on which the cylindric set $A$ depends. Note that $A \subset \tilde{S}^{\prime}$ implies $I=-I$ ). The following proposition characterizes the support of $\mu_{\sigma}$.

Proposition 5.1. Consider the sets:

$$
\begin{aligned}
& \mathscr{A}_{\sigma}=\left\{\hat{u} \in \tilde{S}^{\prime} \mid \lim _{k \rightarrow \infty} \inf \frac{\left|\hat{u}_{\mathbf{k}}\right|^{2} k^{2}}{\sigma^{2} \log k^{2}}=0\right\} \\
& \overline{\mathscr{A}}_{\sigma}=\left\{\hat{u} \in \tilde{S}^{\prime} \mid \lim _{k \rightarrow \infty} \sup \frac{\left|\hat{u}_{\mathbf{k}}\right|^{2} k^{2}}{\sigma^{2} \log k^{2}}=1\right\} .
\end{aligned}
$$

The support of the measure $\mu_{\sigma}$ is contained in the set $\mathscr{A}_{\sigma}=\mathscr{A}_{\sigma} \cap \overline{\mathscr{A}}_{\sigma}$. Moreover energy is infinite $\mu_{\sigma}$-a.e.

Proof. First of all we show that $\mu_{\sigma}\left(\tilde{s}^{\prime} \backslash \mathscr{A}_{\sigma}\right)=0$. We have

$$
\tilde{s}^{\prime} \backslash \mathscr{A}_{\sigma}=\bigcup_{n=1}^{\infty} \bigcup_{N=1}^{\infty} \mathscr{A}_{\sigma}^{(n, N)}
$$

where

$$
\mathscr{A}_{\sigma}^{(n, N)}=\left\{\left.\hat{u} \in \tilde{S}^{\prime}\left|\inf _{k>N}\right| \hat{u}_{\mathbf{k}}\right|^{2} k^{2} /\left(\sigma^{2} \log k^{2}\right) \geqq \frac{1}{n}\right\} .
$$

With the help of formula (5.2) we get:

$$
\mu_{\sigma}\left(\mathscr{A}_{\sigma}^{(n, N)}\right)=\left(\prod_{k \geqq N} \exp \left(-\log k^{2} / n\right)^{1 / 2},\right.
$$

and the conclusion follows from the fact that the infinite product is zero for all $n, N$ since the series $\sum_{k \geqq N}\left(1-1 / k^{2 / n}\right)$ diverges. Consider now the set

$$
\overline{\mathscr{A}}_{\sigma, c}=\left\{\left.\hat{u} \in \hat{S}^{\prime}\left|\lim _{k \rightarrow \infty} \sup \right| \hat{u}_{\mathbf{k}}\right|^{2} k^{2} /\left(\sigma^{2} \log k^{2}\right)<c\right\} .
$$

We have

$$
\overline{\mathscr{A}}_{\sigma, c}=\bigcup_{n=1}^{\infty} \bigcup_{N=1}^{\infty} \overline{\mathscr{A}}_{\sigma, c}^{(n, N)},
$$


where

and

$$
\overline{\mathscr{A}}_{\sigma, c}^{(n, N)}=\left\{\left.\hat{u} \in \tilde{S}^{\prime}\left|\sup _{k \geqq N}\right| \hat{u}_{\mathbf{k}}\right|^{2} k^{2} /\left(\sigma^{2} \log k^{2}\right)<c-1 / n\right\},
$$

$$
\mu_{\sigma}\left(\overline{\mathscr{A}}_{\sigma, c}\right)=\lim _{n \rightarrow \infty} \lim _{N \rightarrow \infty} \mu_{\sigma}\left(\overline{\mathscr{A}}_{\sigma, c}^{(n, N)}\right)
$$

since $\overline{\mathscr{A}}_{\sigma, c}^{(n, N)} \supset \overline{\mathscr{A}}_{\sigma, c}^{(n, N-1)}$ and $\overline{\mathscr{A}}_{\sigma, c}^{(n, N)} \supset \overline{\mathscr{A}}_{\sigma, c}^{(n-1, N)}$. Proceeding as before we find

$$
\mu_{\sigma}\left(\overline{\mathscr{A}}_{\sigma, c}^{(n, N)}\right)=\left(\prod_{k \geqq N}\left(1-k^{-2(c-1 / n)}\right)\right)^{1 / 2}
$$

and the infinite product is zero or finite according to whether the series $\sum_{k \geqq N} k^{-2(c-1 / n)}$ diverges or converges. For $c \leqq 1$ the series always diverges. For $c>1$ the series converges for $n$ so large that $c-1 / n>1$, and for all such $n$ we have

$$
\lim _{N \rightarrow \infty} \mu_{\sigma}\left(\overline{\mathscr{A}}_{\sigma, c}^{(n, N)}\right)=1
$$

whence.it follows easily that $\mu_{\sigma}\left(\overline{\mathscr{A}}_{\sigma}\right)=1$, and therefore $\mu_{\sigma}\left(\mathscr{A}_{\sigma}\right)=1$.

To prove the second assertion we first calculate the average value of the energy:

$$
\mathscr{E}\{E\}=\mathscr{E}\left\{\frac{1}{2} \sum_{\mathbf{k} \in \overline{\mathbb{Z}}^{2}}\left|\hat{u}_{\mathbf{k}}\right|^{2}\right\}=\frac{1}{2} \sigma^{2} \sum_{\mathbf{k} \in \overline{\mathbb{Z}}^{2}} k^{-2}=+\infty .
$$

Consider the sequence of functions

since

$$
: E^{(N)}(\hat{u}):=\frac{1}{2} \sum_{k \leqq N}\left(\left|\hat{u}_{\mathbf{k}}\right|^{2}-\sigma^{2} / k^{2}\right) \quad N=1,2, \ldots
$$

$$
\left\|: E^{(N+1)}(\hat{u}):-: E^{(N)}(\hat{u}):\right\|^{2}\left(d \mu_{\sigma}\right)=\sigma^{4} \sum_{N<k \leqq N+1} k^{-4},
$$

reasoning like in the proof of Theorem 4.2 we conclude that sequence $: E^{(N)}(\hat{u})$ : converges, as $N \rightarrow \infty$, in $L^{2}\left(d \mu_{\sigma}\right)$ as well as almost averywhere. Since the limit is $\mu_{\sigma}$-a.e. finite and the series $\sum_{\mathbf{k} \in \tilde{\mathbb{Z}}^{2}} k^{-2}$ diverges, we conclude that the series $\sum_{\mathbf{k} \in \overline{\mathbb{Z}}^{2}}\left|\hat{u}_{\mathbf{k}}\right|^{2}$ diverges $\mu_{\sigma}$-a.e.

Gaussian equilibrium states are associated to enstrophy conservation : formula (5.2) shows that they are Gibbs states with Gibbs factor $\exp \left(-S / \sigma^{2}\right)\left(\sigma^{2}\right.$ plays here the role of a "temperature" associated to enstrophy). It is natural to take energy conservation into account and to consider equilibrium states with Gibbs factor $\exp \left(-E / \alpha^{2}-S / \sigma^{2}\right)$ (cf. [1]). A standard way to give a mathematical definition of such states is to consider the sequence of the measures on $\tilde{s}^{\prime}$ which have as densities with respect to $d \mu_{\sigma}$ the following cylindric functions:

$$
\varrho_{N}(\hat{u})=\exp \left(-\frac{1}{2} \alpha^{-2} \sum_{k \leqq N}\left|\hat{u}_{\mathbf{k}}\right|^{2}\right) / \int_{\tilde{s}^{\prime}} \exp \left(-\frac{1}{2} \alpha^{-2} \sum_{k \leqq N}\left|\hat{u}_{\mathbf{k}}\right|^{2}\right) d \mu_{\sigma} \quad N=1,2 \ldots
$$

The limit, as $N \rightarrow \infty$, of such measures, if it exists, will define the state we are looking for. 
Theorem 5.1. The sequence of functions (5.4) is a Cauchy sequence in $L^{p}\left(d \mu_{\sigma}\right)$ for any $p \geqq 1$.

Proof. To simplify notations we assume $\sigma=\alpha=1$, and $\mu_{1}=\mu$. We have

$$
\varrho_{N}(\hat{u})=\exp \left(-\frac{1}{2}: E^{(N)}(\hat{u}):\right) / \int_{\tilde{s}^{\prime}} \exp \left(-\frac{1}{2}: E^{(N)}(\hat{u}:) d \mu\right.
$$

$: E^{(N)}(\hat{u})$ : being defined by equality (5.3). The normalization factors

$$
c_{N}=\int_{\widetilde{s}^{\prime}} \exp \left(-\frac{1}{2}: E^{(N)}(\hat{u}):\right) d \mu=\left(\prod_{k \leqq N} \exp \left(k^{-2}-\log \left(1+k^{-2}\right)\right)\right)^{1 / 2}
$$

converge to a finite limit as $N \rightarrow \infty$. Now, since

$$
|\exp (x)-\exp (y)| \leqq|x-y| \cdot|\exp (x)+\exp (y)|
$$

we have, setting $U^{(N)}=-: E^{(N)}$ :

$$
\begin{aligned}
& \left\|\exp \left(U^{(N)}\right)-\exp \left(U^{\left(N^{\prime}\right)}\right)\right\|_{L^{p}(d \mu)}<\left\|U^{(N)}-U^{\left(N^{\prime}\right)}\right\|_{L^{q}(d \mu)} \\
& \cdot\left(\left\|\exp \left(U^{(N)}\right)\right\|_{L^{r}(d \mu)}+\left\|\exp \left(U^{\left(N^{\prime}\right)}\right)\right\|_{L^{r}(d \mu)}\right)
\end{aligned}
$$

for any choice of the integers $r, q, p \geqq 1$ such that $p^{-1}=q^{-1}+r^{-1}$. Moreover since

$$
\left(\left\|\exp \left(U^{(N)}\right)\right\|_{L^{r}(d \mu)}\right)^{r}=\int_{\tilde{S}^{\prime}} \exp \left(r U^{(N)}\right) d \mu=\left(\prod_{k \leqq N} \exp \left(r / k^{-2}-\log \left(1+r / k^{-2}\right)\right)\right)^{1 / 2},
$$

and the series $\sum_{\mathbf{k} \in \overline{\mathbb{Z}}^{2}} r / k^{-2}-\log \left(1+r / k^{-2}\right)$ converges, we have that $\left\|\exp \left(U^{(N)}\right)\right\|_{L^{r}(d \mu)}$ is limited, uniformly in $N$. To estimate $\left\|U^{(N)}-U^{\left(N^{\prime}\right)}\right\|_{L^{q}(d \mu)}$ assume $N^{\prime}>N$ and $q$ to be even (if $q$ is odd we can use the inequality

$$
\left.\left\|U^{(N)}-U^{\left(N^{\prime}\right)}\right\|_{L^{q}(d \mu)} \leqq\left\|U^{(N)}-U^{\left(N^{\prime}\right)}\right\|_{L^{q+1}(d \mu)}\right) .
$$

Setting $q=2 s$ we find:

$$
\begin{aligned}
& \left\|\mathrm{U}^{\left(N^{\prime}\right)}-\mathrm{U}^{(N)}\right\|_{L^{2 s}(d \mu)}^{2 s}=2^{-4 s} \int_{\tilde{s}^{\prime}}\left(\sum_{N<k \leqq N^{\prime}}\left(\left|\hat{u}_{\mathbf{k}}\right|^{2}-1\right) / k^{2}\right)^{2 s} d \mu \\
& =\sum_{m=1}^{s} \sum_{\begin{array}{r}
p_{1}, \ldots, p_{m}=0 \\
p_{1}>p_{2}>\ldots>p_{m} \\
p_{1}+p_{2}+\ldots+p_{m}=s
\end{array}}^{s} n\left(p_{1}, \ldots, p_{m}\right) \sum_{\mathbf{k}_{1} \neq \ldots \neq \mathbf{k}_{m} \in I_{N}+1} k_{1}^{-4 p_{1}} \ldots k_{m}^{-4 p_{m}}
\end{aligned}
$$

where $n\left(p_{1}, \ldots, p_{m}\right)$ are combinatorial factors, so that the result follows immediately.

As a consequence of Theorem 5.1 we have a new class of gaussian measures which are absolutely continuous with respect to the old ones, with density given by the limits of sequences (5.4). Therefore they have the same support properties. The new class is labelled by two positive parameters, $\sigma$ and $\alpha$. It is easy to show that the stationary Hopf equation makes sense for such measures, and that they are stationary solution of such equation. In fact the characteristic functionals are

$$
\Phi_{\alpha, \sigma}(\hat{\theta})=\exp \left(-\frac{1}{2} \sigma^{2} \sum_{\mathbf{k} \in \overline{\mathbb{Z}}^{2}}\left|\hat{\theta}_{\mathbf{k}}\right|^{2} /\left(k^{2} \sigma^{-2}+\alpha^{-2}\right)\right)
$$

and the proof of Theorem 4.2 can be repeated step to step. We have found therefore a new class of gaussian equilibrium states. We remark that similar results on gaussian states are contained in the paper [18]. 


\section{Poissonian States}

Definition 5.1. We shall call "simply poissonian equilibrium state with vortex strength $\kappa$ and parameter $c "(\kappa \neq 0$ and $c>0$ are real numbers $)$ the equilibrium state of the class described by Theorem 4.1 which corresponds to the function

$$
\psi(t)=c(\exp (i \kappa t)-1)
$$

For such a state vorticity is distributed according to the characteristic functional

$$
\chi_{\psi}(\phi)=\exp \left(\int_{T^{2}} c(\exp (i \kappa \phi(x))-1) d x\right) \quad \phi \in \mathscr{S}\left(T^{2}\right) .
$$

Definition 5.2. We shall call "poissonian equilibrium state" any state of the class described by Theorem 4.1 which corresponds to a function $\psi$ of the type $\psi(t)=\sum_{j=1}^{n} c_{j}\left(\exp \left(i \kappa_{j} t\right)-1\right), n$ being a positive integer, and $c_{j}>0, \kappa_{j} \neq 0$ real numbers. For such states the vorticity distribution is a superposition of a finite number of independent simply poissonian vorticity distribution.

Proposition 5.2. For any poissonian state there exists a stochastic measure (with sign) $\xi_{\psi(\cdot)}$ on $T^{2}$, with finite total variation $\mu_{\psi}$-a.e., such that the g.r.f. which gives the vorticity distribution admits $\mu_{\psi}$-a.e. the representation

$$
\langle\xi, \phi\rangle=\int_{T^{2}} \phi(x) \xi_{\psi}(d x) \quad \phi \in \mathscr{S}\left(T^{2}\right) .
$$

Proof. Since this result is a consequence of a general theorem which can be found in [16] (for the case of processes, but the restriction is not essential), we shall only outline the proof for simply poissonian states (the generalization to poissonian states is however very simple). Consider the g.r.f. identified by the characteristic functional (5.5), with $c=\kappa=1$ for simplicity). The latter obviously makes sense for $\phi=t \chi_{A}$, with $A$ a Borel set in $T^{2}$ and $\chi_{A}$ its characteristic function, so that we can associate to any Borel set $A \subset T^{2}$ a random variable $\xi_{\psi}^{\prime}(A)$ such that

$$
\mathscr{E}_{\psi}\left\{\xi_{\psi}^{\prime}(A)\right\}=m(A), \quad \mathscr{E}_{\psi}\left\{\xi_{\psi}^{\prime}(A) \xi_{\psi}^{\prime}(B)\right\}=m(A)+m(B)+m(A \cap B)
$$

[where $m(\cdot)$ is the Haar measure on $T^{2}$ and $m\left(T^{2}\right)=(2 \pi)^{2}$ ]. Now if we associate to any Borel set $A \subset T^{2}$ the random variable $\xi_{\psi}(A)=\xi_{\psi}^{\prime}(A)-m(A)$ it is easy to see that:

i) $\xi_{\psi}(A) \in L^{2}\left(d \mu_{\psi}\right)$

ii) $\xi_{\psi}(A \cup B)=\xi_{\psi}(A)+\xi_{\psi}(B) \quad \mu_{\psi}$-a.e. if $A \cap B=\emptyset$

iii) $\mathscr{E}_{\psi}\left(\xi_{\psi}(A) \xi_{\psi}(B)\right)=m(A \cap B)$

for any Borel sets $A, B \subset T^{2}$. The family of random variables $\xi_{\psi}(A)$, for all Borel sets $A$, defines therefore a stochastic measure on $T^{2}$, whose structure function is $m(\cdot)$, and a trivial verification shows that representation (5.6) takes place $\mu_{\psi}$-a.e. Note that for any Borel set $A \xi_{\psi}(A)+m(A)$ is a random variable which takes integer values $\mu_{\psi}$-a.e. and is Poisson distributed with parameter $m(A)$.

Corollary 5.1. For any poissonian equilibrium state energy is infinite $\mu_{\psi}$-a.e.

Proof. Again we will give the proof only for the simply poissonian state with $c=\kappa=1$, since the extension is not difficult. As a consequence of Proposition 5.1 
we have that $\mu_{\psi}$-a.e. $\hat{u}_{\mathbf{k}}=-\frac{i}{k} \sum_{j=1}^{n} \exp \left(i \mathbf{k} \cdot \mathbf{x}_{j}\right)$, the finite positive integer $n$ and the points $\mathbf{x}_{j} \in T^{2}\left(\mathbf{x}_{i} \neq \mathbf{x}_{j}\right.$ for $\left.i \neq j\right)$ depending on $\hat{u}$. Therefore:

$$
\sum_{\mathbf{k} \in \tilde{\mathbb{Z}}^{2}}\left|\hat{u}_{\mathbf{k}}\right|^{2}=\sum_{\mathbf{k} \in \tilde{\mathbb{Z}}^{2}} k^{-2}\left(n+\sum_{i<j} \cos \mathbf{k} \cdot\left(\mathbf{x}_{i}-\mathbf{x}_{j}\right)\right),
$$

and the result follows immediately since the series $\sum_{\mathbf{k} \in \overline{\mathbb{Z}}^{2}} k^{-2}$ diverges, whereas the series defining the Green's function of the Laplacian in $T^{2}, \sum_{\mathbf{k} \in \overline{\mathbb{Z}}^{2}} k^{-2} \exp (i \mathbf{k} \cdot \mathbf{x})$,
converges for $\mathbf{x} \neq 0$.

\section{Equilibrium Dynamics for a Class of Poissonian States}

In this paragraph we construct the time evolution for the Euler equation on a set of full measure with respect to all poissonian states for which vorticity takes only positive, or only negative, values (i.e. for which all vortices rotate in the same direction).

First of all we need to give sense the Euler equation (2.12) as a differential equation in $\tilde{s}^{\prime}$.

Definition 6.1. We shall say that the function $\hat{u}(\cdot): \mathbb{R}^{1} \rightarrow \tilde{s}^{\prime}$ is a solution of the Euler equation with initial data $\hat{u}_{0}$ whenever $\hat{u}(0)=\hat{u}_{0}$, the limit $B(\hat{u}(t))=\lim _{N \rightarrow \infty} B^{(N)}(\hat{u}(t))$ exists in $\tilde{s}^{\prime}$, for all $t$, the functions $\langle\hat{u}(t), \hat{\theta}\rangle$ are derivable for all $t$ and all $\hat{\theta} \overrightarrow{\hat{\theta} \in \tilde{s}}$, and the equality takes place

$$
\frac{d}{d t}\langle\hat{u}(t), \hat{\theta}\rangle=\langle B(\hat{u}), \hat{\theta}\rangle \quad t \in \mathbb{R}^{1}, \quad \hat{\theta} \in \tilde{s} .
$$

In the following we shall call "Euler equation" Eq. (6.1). It is easily seen (cf. [11]) that whenever $\sum_{\mathbf{k} \in \overline{\mathbb{Z}}^{2}} k^{2}\left|\hat{u}_{\mathbf{k}}^{(0)}\right|^{2}<\infty$ Eq. (6.1) is equivalent to the usual Euler equation in weak form.

Theorem 6.1. Let $\mu_{\psi}$ be the measure on $\tilde{s}^{\prime}$ corresponding to a simply poissonian state. There exists a set $P_{\psi}, \mu_{\psi}\left(P_{\psi}\right)=1$, such that for any $u \in P_{\psi}$ there is a solution $\hat{u}(\cdot)$ of the Euler equation (6.1) satisfying the initial condition $\hat{u}(0)=\hat{u}$. The solution is such that the components $\hat{u}_{\mathbf{k}}(t)$ are analytic in $t$, and is unique within the class of functions possessing this property (hereafter to be called "analytic").

Proof. We set for simplicity $\kappa=1$. Consider the sets

$$
\begin{aligned}
P_{\psi}^{(n)}= & \left\{\hat{u} \in \tilde{s}^{\prime} \mid \hat{u}_{\mathbf{k}}=(i k)^{-1} \sum_{j=1}^{n} \exp \left(i \mathbf{k} \cdot \mathbf{x}_{j}\right)\right. \\
& \text { for some } \left.\left(\mathbf{x}_{1}, \ldots, \mathbf{x}_{n}\right) \in\left(T^{2}\right)^{n}, \mathbf{x}_{i} \neq \mathbf{x}_{j} \text { for } i \neq j\right\} \quad n=1,2, \ldots
\end{aligned}
$$

As a consequence of Proposition 5.2 it is easily seen that the set $P_{\psi}=\bigcup_{n=1}^{\infty} P_{\psi}^{(n)}$ is a set of full measure: $\mu_{\psi}\left(P_{\psi}\right)=1$. Consider now the ordinary differential equation in $\left(T^{2}\right)^{n}$

$$
\frac{d}{d t} \mathbf{x}_{i}(t)=\operatorname{rot}_{\mathbf{x}_{i}} \sum_{j \neq 1} g\left(\mathbf{x}_{i}(t)-\mathbf{x}_{j}(t)\right) \quad i=1, \ldots, n
$$


with the initial condition

$$
\mathbf{x}_{i}(0)=\mathbf{x}_{i}
$$

$g$ being the Green's function associated to the operator $-\Delta$. Equations (6.2) are a set of hamiltonian equations, the hamiltonian being

$$
\mathscr{H}=\sum_{i \neq j} g\left(\mathbf{x}_{i}-\mathbf{x}_{j}\right)
$$

(they are the Onsager vortex equations on $T^{2}$, cf. [2]). Since the function $g(\mathbf{x})$ is everywhere analytic except at $\mathbf{x}=0$, where it diverges logarithmically to $+\infty$, the variety $\mathscr{H}=$ const in $\left(T^{2}\right)^{n}$ keeps everywhere at a finite distance (depending on $\mathscr{H}$ ) from the hyperplanes $\mathbf{x}_{i}=\mathbf{x}_{j}, i \neq j$, so that (6.2), as a differential equation with analytic right hand side posseses a unique analytic solution satisfying the initial condition (6.3). Denote by $\mathbf{x}_{i}(t), i=1, \ldots, n$, the solution and consider the function

$$
\hat{u}(t)=\left\{(i k)^{-1} \sum_{j=1}^{n} \exp \left(i \mathbf{k} \cdot \mathbf{x}_{j}(t)\right)\right\}_{\mathbf{k} \in \overline{\mathbb{Z}}^{2}} .
$$

$\langle\hat{u}(t), \hat{\theta}\rangle$ is differentiable in $t$ for any $t \in \mathbb{R}^{1}$ and we have

$$
\frac{d}{d t}\langle\hat{u}(t), \hat{\theta}\rangle=\sum_{\mathbf{k} \in \overline{\mathbb{Z}}^{2}} \frac{d}{d t} \overline{\hat{u}}_{\mathbf{k}}(t) \hat{\theta}_{\mathbf{k}}=\sum_{\mathbf{k} \in \overline{\mathbb{Z}}^{2}} \frac{\mathbf{k}}{k} \cdot \sum_{j=1}^{n} \mathbf{x}_{i} \exp \left(-i \mathbf{k} \cdot \mathbf{x}_{j}\right) \hat{\theta}_{\mathbf{k}} .
$$

A straightforward calculation yields

$$
\begin{aligned}
B_{\mathbf{k}}^{(N)}(\hat{u}(t))= & -i \sum_{\substack{\mathbf{m} \in I_{N} \\
\mathbf{k}-\mathbf{m} \in I_{N}}}\left[\left(\mathbf{m}^{\perp} \cdot \mathbf{k}\right)\left(|\mathbf{k}-\mathbf{m}|^{2}-m^{2}\right) / 2 k|\mathbf{k}-\mathbf{m}| m\right] \hat{u}_{\mathbf{m}}(t) \hat{u}_{\mathbf{k}-\mathbf{m}}(t) \\
= & -(i / k) \sum_{\substack{\mathbf{m} \in I_{N} \\
\mathbf{k}-\mathbf{m} \in I_{N}}}\left(\left(\mathbf{m}^{\perp} \cdot \mathbf{k}\right)|\mathbf{k}-\mathbf{m}| / m\right) \hat{u}_{\mathbf{m}}(t) \hat{u}_{\mathbf{k}-\mathbf{m}}(t) \\
= & (i / k) \sum_{\substack{\mathbf{m} \in I_{N} \\
\mathbf{k}-\mathbf{m} \in I_{N}}}\left(\mathbf{m}^{\perp} \cdot \mathbf{k} / m^{2}\right) \cdot \sum_{s, j=1}^{n} \exp \left(i\left(\mathbf{m} \cdot \mathbf{x}_{j}(t)+(\mathbf{k}-\mathbf{m}) \cdot \mathbf{x}_{s}(t)\right)\right) \\
= & (i \mathbf{k} / k) \cdot \sum_{s=1}^{n} \exp \left(i \mathbf{k} \cdot \mathbf{x}_{s}(t)\right) \sum_{j \neq s} \sum_{\substack{\mathbf{m} \in I_{N} \\
\mathbf{k}-\mathbf{m} \in I_{N}}}\left(\mathbf{m}^{\perp} / m^{2}\right) \exp \left(i \mathbf{m} \cdot\left(\mathbf{x}_{j}(t)-\mathbf{x}_{s}(t)\right)\right) \\
& +(i / k) \sum_{s=1}^{n} \exp \left(i \mathbf{k} \cdot \mathbf{x}_{s}(t)\right) \sum_{\substack{\mathbf{m} \in I_{N} \\
\mathbf{k}-\mathbf{m} \in I_{N}}} \mathbf{m}^{\perp} \cdot \mathbf{k} / m^{2} .
\end{aligned}
$$

Since

$$
\sum_{\substack{\mathbf{m} \in I_{N} \\ \mathbf{k}-\mathbf{m} \in I_{N}}} \mathbf{m}^{\perp} \cdot \mathbf{k} / m^{2}=\sum_{\substack{\mathbf{m} \in I_{N} \\ \mathbf{k}-\mathbf{m} \in I_{N}}} \frac{1}{2} \mathbf{m}^{\perp} \cdot \mathbf{k}\left(k^{2}-2 \mathbf{k} \cdot \mathbf{m}\right) /\left(m^{2}|\mathbf{k}-\mathbf{m}|^{2}\right)=0
$$

(cf. proof of Theorem 4.2), and since

$$
\sum_{\substack{\mathbf{m} \in I_{N} \\ \mathbf{k}=\mathbf{m} \in I_{N}}}\left(\mathbf{m}^{\perp} / m^{2}\right) \exp \left(i \mathbf{m} \cdot\left(\mathbf{x}_{j}(t)-\mathbf{x}_{s}(t)\right)\right) \underset{N \rightarrow \infty}{\longrightarrow} i \operatorname{rot}_{\mathbf{x}_{s}} g\left(\mathbf{x}_{j}(t)-\mathbf{x}_{s}(t)\right),
$$

we have that $\frac{d}{d t} \hat{u}_{\mathbf{k}}(t)=B_{\mathbf{k}}(\hat{u}(t))$ and $(6.1)$ is satisfied. The functions $\hat{u}_{\mathbf{k}}(t)$ are obviously analytic since a composition of analytic functions is analytic. Let $\hat{v}(t)$ be another 
analytic solution satisfying the same initial condition at $t=0$; the functions $\tilde{v}_{\mathbf{k}}$ coincide with the functions $\hat{u}_{\mathbf{k}}$ and so do all their derivatives, which may calculated with the help of Eq. (6.2) in terms of the initial data. Since the function $\hat{v}_{\mathbf{k}}(t)$ and $\hat{u}_{\mathbf{k}}(t)$ are analytic and have all derivaties equal at $t=0$, they must coincide.

Actually a stronger result is true, which we give as a corollary.

Corollary 6.1. There exists a set $P$ in $\tilde{s}^{\prime}$ such that for any $\hat{u} \in P$ there is a unique analytic solution $\hat{u}(\cdot)$ of the Euler equation (6.1) satisfying the initial condition $\hat{u}(0)=\hat{u}$, and, moreover, $\mu_{\psi}(P)=1$ for any measure $\mu_{\psi}$ corresponding to a simply poissonian state or to a poissonian state for which all vortex strengths $\kappa_{j}$ are of the same sign.

Proof. Consider the sets

$$
\begin{aligned}
P^{(n)}= & \left\{\hat{u} \in \tilde{s}^{\prime} \mid \hat{u}_{\mathbf{k}}=(i k)^{-1} \sum_{j=1}^{n} \kappa_{j} \exp \left(i \mathbf{k} \cdot \mathbf{x}_{j}\right),\right. \\
& \text { for some } \left.\left(\mathbf{x}_{1}, \ldots, \mathbf{x}_{n}\right) \in\left(T^{2}\right)^{n}, \kappa_{s}, \kappa_{j}>0 \text { and } \mathbf{x}_{j} \neq \mathbf{x}_{s} \text { for } s \neq j\right\} \quad n=1,2, \ldots
\end{aligned}
$$

The set $P^{(n)}$ corresponds to all possible configurations of $n$ vortices with vortex strengths of the same sign (i.e. all vortices rotate in the same direction). It is not hard to see (cf. Proposition 5.2) that the set $P=\bigcup_{n=1}^{\infty} P^{(n)}$ is a set of full measure for any poissonian state satisfying the conditions of the corollary. Now, by repeating, with small modifications, the proof of Theorem 6.1 it is seen that existence of dynamics on the set $P$ follows from the existence of a unique solution for finite systems of vortices rotating in the same direction.

Notice that for poissonian states with vortex strengths of both signs the theorem does not hold, since finite configurations of vortices of both signs may be catastriphic, i.e. two vortices may collapse in a finite time [17].

\section{Conclusions}

Equilibrium dynamics for poissonian states admitting only positive (or negative) vorticity has been easily obtained via the Onsager vortex equations. The problem is more complicated for the other physically interesting cases. For poissonian states with vorticity of both signs equilibrium dynamics can be constructed, by means of the Onsager vortex equations, only of the set of the catastrophic configurations which we mentioned at the end of the preceding paragraph is shown to be of zero equilibrium measure, as it is reasonable to expect. For this, however, one should wait until a sufficiently complete characterization of the catastrophic set is given (which seems to be a hard task). For gaussian states there are some preliminary results [18], however the problem is essentially open.

Once equilibrium dynamics have been constructed we can investigate the evolution of initial states which are absolutely continuous with respect to the equilibrium measure. Convergence to equilibrium for such states is strictly connected to the ergodic properties of the corresponding "equilibrium dynamical system" (which is the dynamical system on phase space given by equilibrium measure and time evolution). The investigation of the ergodic properties of the 
equilibrium states is probably as difficult a problem as it is in statistical mechanics (cf. [19]).

For poissonian states we can say for sure that ergodicity does not hold, since there are invariants of the motion (at least the vortex number, the hamiltonian and the vorticity center $\left.\mathbf{x}=\sum_{i=1}^{n} \mathbf{x}_{i}(t) \kappa_{i}\right)$. Even on the manifolds identified by such integrals of the motion the system is in general nonergodic and apparently a variant of the Kolmogorov-Arnol'd-Moser theorem holds [20]. The situation is similar to that of finite particle systems.

Acknowledgements. We are indepted first of all to G. Gallavotti who stimulated our interest in the subject. We thank also R. L. Dobrushin, E. A. Novikov, A. Pellegrinotti, Ya. G. Sinai, D. Surgailis, L. Triolo and A. M. Yaglom for many useful conversations and comments.

\section{References}

1. Lee, T.D.: Quart. Appl. Math. 10, 69 (1952)

2. Novikov, E.A.: Sov. Phys. JETP 41, 937 (1976)

3. Gurievich, B.M., Suhov, Ju.M.: Commun. Math. Phys. 49, 63 (1976)

4. Dobrushin, R.L., Suhov, Ju.M.: Lecture notes in physics, Vol. 80, p. 325. Berlin, Heidelberg, New York: Springer 1978

5. Fox, D.G., Orszag, S.A.: Phys. Rev. Lett. 16, 169 (1973)

6. Lanford, O.E. III, Commun. Math. Phys. 9, 169 (1968); 11, 257 (1969)

7. Fritz, J., Dobrushin, R.L.: Commun. Math. Phys. 57, 67 (1977)

8. Lanford, O.E.III : Lecture notes in physics, Vol. 38, p. 1. Berlin, Heidelberg, New York: Springer 1975

9. Kato, T.: Arch. Rat. Anal. 25, 188 (1967)

10. Bardos, C.: I. Math. An. Appl. 40, 769 (1972)

11. Boldrighini, C.: Introduzione alla Fluidodinamica, Quaderni del C.N.R., Roma., 1979

12. Lee, J.: Phys. Fluids 20, 1250 (1977)

13. Gelfand, I.M., Vilenkin, M.Y.: Generalized functions, Vol. IV. New York: Academic Press 1964

14. Reed, M., Simon, B.: Functional analysis, Vol. 1. New York: Academic Press 1972

15. Gnedenko, B.V., Kolmogorov, A.M.: Limit distributions for sums of independent random variables. Cambridge: Addison Wesley 1954

16. Skorokhod, A.V.: Stochastic processes with independent increments. Moscow: Nauka 1964

17. Novikov, E.A.: Teoretičeskaja i Matematičeskaja Fizika. To appear

18. Albeverio, S., Ribeiro de Faria, M., Høegh-Krohn, R.: Centre de Physique Teorique, CNRS Marseille Université d'Aix, Marseille II, Uer Scientifique di Luminy, preprint, 1978

19. Goldstein, S., Lebowitz, J.L., Aizenman, M.: Lecture notes in physics, Vol. 38, pp. 112. Berlin, Heidelberg, New York: Springer 1975

20. Sinai, Ya.G.: Private communication

Communicated by J. L. Lebowitz

Received May 21, 1979 